\title{
Effect of Intracisternal Injection of Endothelin-1 on Regional Cerebral Blood Flow in Cats
}

\author{
Tadashi Morimoto, Tetsu HANAMURA*, Tomio SASAKI, \\ Isamu SAITO and Kintomo TAKAKURA
}

\author{
Department of Neurosurgery, Faculty of Medicine, University of Tokyo, Tokyo; \\ ${ }^{*}$ Department of Neurosurgery, Tokyo Metropolitan Police Hospital, Tokyo
}

\begin{abstract}
The effect of endothelin-1 on regional cerebral blood flow ( $\mathrm{CCBF}$ ) was investigated in adult mongrel cats. Endothelin-1 was injected into the cisterna magna and rCBF was measured by the hydrogen clearance method before and every 30 minutes for 180 minutes after the injection. Endothelin-1 $\left(10^{-11}\right.$ $\mathrm{mol}$ and $10^{-9} \mathrm{~mol}$ ) induced a significant decrease in $\mathrm{rCBF}$ and an increase in arterial pressure. The effects of endothelin-1 on rCBF and arterial pressure were mediated, at least in part, by $\mathrm{Ca}^{2+}$, because pretreatment with intracisternally injected nicardipine prevented the changes. Cerebral angiograms obtained before and after endothelin-1 injection demonstrated severe constriction of the basilar artery, but constriction of the internal carotid and middle cerebral arteries was mild. Since the rCBF measured was in the territory of the middle cerebral artery, the decrease in blood flow was probably not solely due to the artery constriction. Other mechanisms such as arteriole constriction and/or regulation of $\mathrm{rCBF}$ by the central nervous system may also occur.
\end{abstract}

Key words: endothelin, regional cerebral blood flow, nicardipine

\section{Introduction}

In 1982, De Mey and Vanhoutte ${ }^{3)}$ showed that arteriovenous constriction in vitro caused by norepinephrine, thrombin, and arachidonic acid is dependent on or enhanced by the endothelium. Anoxic potentiation of norepinephrine-induced vascular contraction is reduced in the absence of endothelium. ${ }^{3,4)}$ Rubanyi and Vanhoutte ${ }^{16)}$ reported that diffusible factors mediate the constriction mechanism. In 1987, O'Brien et al. ${ }^{13)}$ discovered the release of a vasoconstrictive peptide from bovine aorta and pulmonary artery endothelial cells in vitro, and named it endothelium-derived constricting factor. In 1988, Yanagisawa et al. ${ }^{21)}$ isolated a novel vasoconstrictor peptide, endothelin, from the supernatant of porcine aorta endothelium culture, with extremely potent vasoconstrictivity. The human endothelin family consists of three distinct isopeptides. ${ }^{8}$ Endothelin-1 is produced by vascular endothelial cells, and endothelin-3 is possibly a neural form of endothelin. ${ }^{8)}$

To clarify the role of endothelin- 1 in vasospasm following subarachnoid hemorrhage (SAH), we studied the effect of intracisternal injection of endothelin-1 on regional cerebral blood flow ( $\mathrm{rCBF}$ ) in cats.

\section{Materials and Methods}

I. rCBF after intracisternal injection of endothelin-1

Adult mongrel cats of either sex, weighing 2.2-3.8 $\mathrm{kg}$, were anesthetized with halothane and intubated. The right femoral artery was catheterized for continuous recording of systemic arterial pressure and the vein for intravenous injection.

The head was fixed in a stereotactic apparatus and several small burr holes drilled in the left half of the calvarium. The dura was incised and platinum wire electrodes with bared tips $0.5-1.0 \mathrm{~mm}$ long were inserted into the cerebral cortex over the para-

Received May 14, 1990; Accepted February 13, 1991

Author's present address: I. Saito, M.D., Department of Neurosurgery, Kyorin University School of Medicine, Mitaka, Tokyo, Japan. 
Sylvian area using an operating microscope. The electrodes were fixed and the dura closed by cotton balls and alpha-cyanoacrylate (Aron Alpha $\mathrm{A}^{\circledR}$, Sankyo Co., Ltd., Tokyo). A reference $\mathrm{Ag} / \mathrm{AgCl}$ electrode was implanted subcutaneously on the right side. After electrode placement, halothane was discontinued. The animals were immobilized by intravenous gallamine administration and connected to a ventilator. Anesthesia was maintained with $25 \%$ $\mathrm{O}_{2} / 75 \% \mathrm{~N}_{2} \mathrm{O}$, and the respiration adjusted to maintain arterial carbon dioxide partial pressure between 30 and $40 \mathrm{mmHg}$. Arterial blood gases were intermittently analyzed and the end-tidal $\mathrm{CO}_{2}$ and $\mathrm{O}_{2}$ concentrations continuously monitored. About 30 minutes were necessary for the electrode system to become stabilized in the tissue. Hydrogen gas was introduced directly into the endotracheal tube for about 2 minutes $\left(17 \% \mathrm{O}_{2}, 50 \% \mathrm{~N}_{2}, 33 \% \mathrm{H}_{2}\right)$ and the clearance monitored with an amplifier system and a 6-channel pen recorder. rCBF was calculated from the clearance curves obtained. ${ }^{6,15,20)}$

After the control rCBF level was measured twice, a 21-gauge butterfly needle was inserted into the cisterna magna with the neck flexed and $1 \mathrm{ml}$ of cerebrospinal fluid (CSF) withdrawn. Endothelin-1 (Peptide Institute, Inc., Minoo, Osaka) dissolved in $1 \mathrm{ml}$ of $0.1 \mathrm{M}$ phosphate-buffered saline (PBS) was then injected via the same route and the animal maintained in the head-down position for 10 minutes. The cats were divided into following three groups: Group A, $1 \mathrm{ml}$ of PBS without endothelin-1 was injected $(\mathrm{n}=5)$; Group B, the PBS contained $10^{-11} \mathrm{~mol}$ of endothelin-1 $(\mathrm{n}=7)$; and Group $\mathrm{C}, 10^{-9} \mathrm{~mol}(\mathrm{n}=$ 6). The $\mathrm{rCBF}$ was measured every 30 minutes for 180 minutes using 23,30 , and 30 electrodes, respectively. Electrodes causing significant tissue injury or inserted into the white matter as judged by baseline clearance curves were excluded.

\section{Pretreatment with nicardipine}

To investigate the effect of $\mathrm{Ca}^{2+}$ channel blocker on endothelin- 1 induced rCBF changes, $0.5 \mathrm{mg}$ of nicardipine $(0.5 \mathrm{ml})$ (Yamanouchi Pharmaceutical Co., Ltd., Tokyo) was administered through a 21 gauge butterfly needle inserted into the cisterna magna after measurement of the control rCBF level. In four cats, the $\mathrm{rCBF}$ was measured 30 minutes after nicardipine injection, then PBS without endothelin-1 was administered, and $\mathrm{rCBF}$ measurements were repeated every 30 minutes for 180 minutes using 22 electrodes (Group N). In another four cats, $10^{-9} \mathrm{~mol}$ of endothelin- 1 was administered $30 \mathrm{~min}-$ utes after nicardipine injection, and the rCBF measured using 18 electrodes (Group NE).

\section{Cerebral angiography}

Adult mongrel cats were anesthetized and intubated, and the right femoral artery and vein were catheterized as described above. The left common carotid artery was catheterized for angiography. The head was fixed in a stereotactic apparatus and the animals were immobilized and ventilated. Angiograms were obtained before and after endothelin-1 injection at predetermined time intervals (before, 30 , 60,120 , and 180 minutes after injection). Endothelin1 doses given were the same as above ( 0 ; control, $10^{-11} \mathrm{~mol}$, and $\left.10^{-9} \mathrm{~mol}\right)$.

\section{Statistical analysis}

Data were presented as mean \pm SEM. rCBF changes in the groups were compared using analysis of variance. Differences in $\mathrm{rCBF}$ at the same time between Groups A, B, and $\mathrm{C}$ were evaluated by analysis of variance and Scheffe's test. Differences in rCBF between Groups N and NE were evaluated by the unpaired Student's t-test. rCBF differences before and after endothelin-1 injection were evaluated using the paired Student's t-test. Differences at the $p<0.05$ level were considered statistically significant.

\section{Results}

\section{I. rCBF after intracisternal injection of endothelin-} 1 (Fig. 1)

In Group A, rCBF did not change after PBS injection. However, intracisternal injection of endothelin-1 resulted in a significant decrease in $\mathrm{rCBF}$ from 30 to 180 minutes after injection in Groups B and $\mathrm{C}(\mathrm{p}<0.01)$. In Group B, rCBF 180 minutes after endothelin-1 injection was $82.6 \pm 3.6 \mathrm{ml} / 100$ $\mathrm{gm} / \mathrm{min}$, about $78 \%$ of the baseline value, and significantly lower than that in Group A $(p<0.05)$. In Group $C$, in which the dose of endothelin-1 was higher, the rCBF at 180 minutes was about $53 \%$ of the baseline value. All rCBF values from 30 to 180 minutes in Group $C$ were significantly lower than those in Group A $(p<0.001)$.

\section{Pretreatment with nicardipine (Fig. 2)}

rCBF increased 30 minutes after intracisternal injection of nicardipine $(p<0.01)$, and thereafter decreased gradually in both groups. Although the secondary flow reduction tended to be larger in Group $\mathrm{NE}$, no significant differences were found.

\section{Systemic arterial pressure}

In Group A, the systemic arterial pressure remained unchanged after intracisternal PBS injection. The 


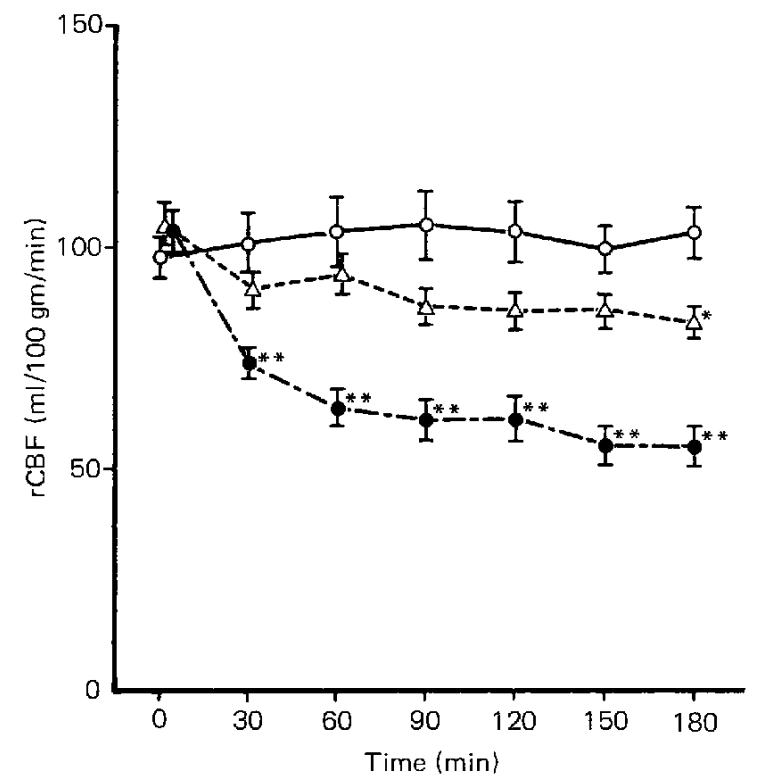

Fig. 1 rCBF before and after intracisternal injection of endothelin-1. $O$ : Group $A$ (control), $\triangle$ : Group B $\left(10^{-11} \mathrm{~mol}\right), \bullet$ : Group C $\left(10^{-9} \mathrm{~mol}\right)$. ${ }^{*} \mathrm{p}<0.05,{ }^{* *} \mathrm{p}<0.001$. Reproduced with permission from ref. 11.

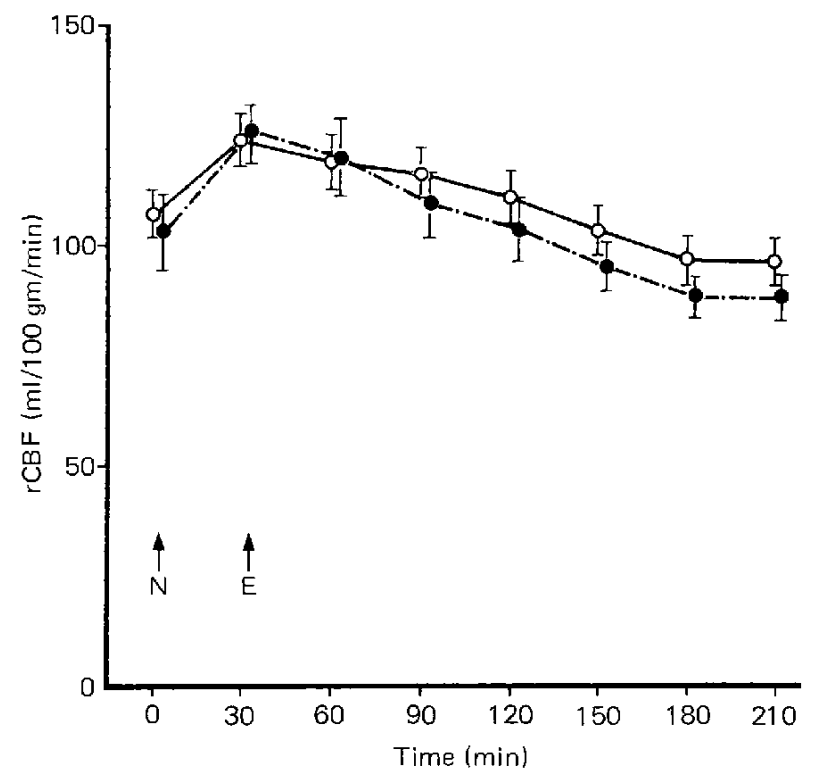

Fig. 2 rCBF before and after intracisternal injection of nicardipine $(\mathrm{N})$ and endothelin-l $(\mathrm{E}) . \quad \mathrm{O}$ : Group N (nicardipine $0.5 \mathrm{mg}$ ), $\bullet$ : Group $\mathrm{NE}$ (nicardipine $0.5 \mathrm{mg}+$ endothelin-1 $10^{-9} \mathrm{~mol}$ ). No statistically significant difference is found between the two groups. Reproduced with permission from ref. 11 . arterial pressure increased after endothelin-1 injection in four animals in Group B and five in Group C. It decreased in only one animal in Group B. Intracisternal endothelin-1 injection produced no changes in arterial pressure in two animals in Group B and one in Group C (Figs. 3 and 4).

Intracisternal nicardipine injection caused a slight decrease in arterial pressure. Pretreatment with nicardipine inhibited the increase in arterial pressure produced by the intracisternal endothelin- 1 injection in all animals (Fig. 5).

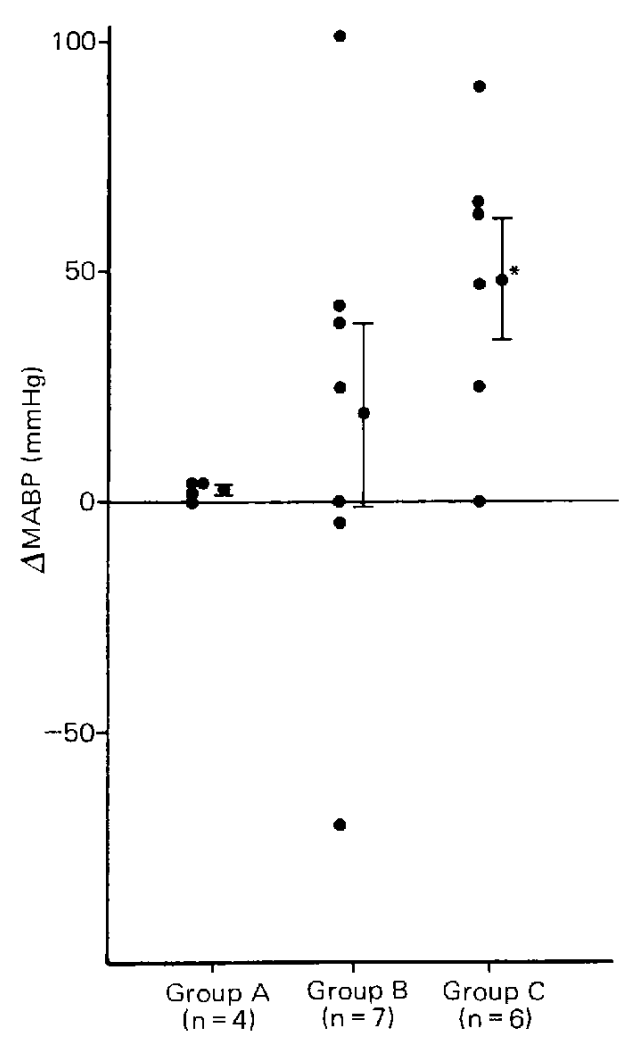

Fig. 3 Changes in mean arterial blood pressure (MABP) after intracisternal injection of endothelin-1 or the vehicle. ${ }^{*} p<0.05$ vs. Group A.

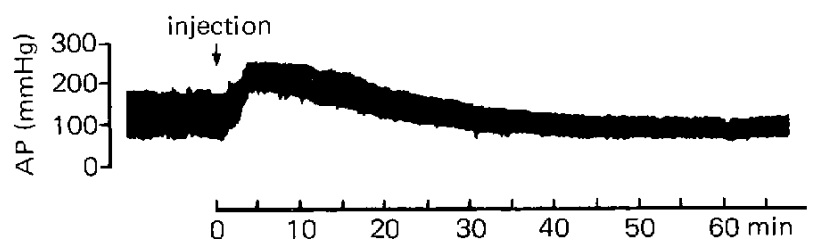

Fig. 4 Typical recording of arterial pressure (AP) before and after intracisternal injection of endothelin-1 $\left(10^{-9} \mathrm{~mol}\right)$. 


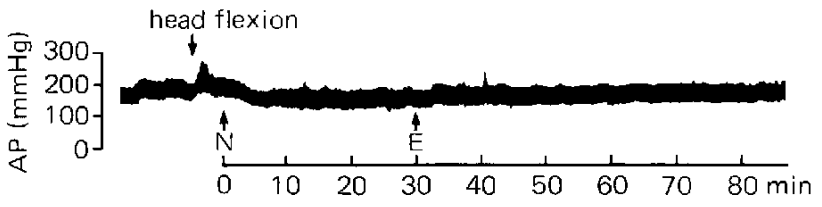

Fig. 5 Typical recording of arterial pressure (AP) before and after intracisternal injection of nicardipine $(0.5 \mathrm{mg}) \quad(\mathrm{N})$ and endothelin-l $\left(10^{-9} \mathrm{~mol}\right)(\mathrm{E})$.

\section{Cerebral angiography}

Intracisternal endothelin-1 injection caused severe basilar artery (BA) constriction. In Group B, the diameters of the BA 60 and 120 minutes after the injection were $71 \%$ and $71 \%$ of the baseline value, and in Group C, $52 \%$ and $62 \%$, respectively. However, internal carotid (ICA) and middle cerebral arteries (MCA) constriction was much less severe ( $89 \%$ and $92 \%$ of the baseline diameter at 60 and 120 minutes after the injection in Group B, and $84 \%$ and $80 \%$ in Group C, respectively). The BA constriction lasted for more than 3 hours (Figs. 6 and 7).

\section{Discussion}

The present study demonstrated that intracisternal injection of endothelin-1 significantly decreased $\mathrm{rCBF}$ and increased arterial pressure in cats. These changes were mediated, at least in part, by $\mathrm{Ca}^{2+}$, because they were prevented by the nicardipine pretreatment.

Endothelin-1 has a potent vasoconstrictor effect in vitro on porcine coronary artery, rat aorta, cat BA, rabbit mesenteric artery, dog mesenteric, femoral and renal arteries, human mesenteric and pulmonary artery branches, ${ }^{21}$ and dog, rabbit and monkey cerebral arteries. ${ }^{12}$ The endothelin-induced contraction in monkey MCA was very long-lasting. About $70 \%$ of maximum contractile response to endothelin at $3 \times 10^{-9} \mathrm{M}$ was still present 12 hours after endothelin administration. ${ }^{2)}$ In vivo experiments have also shown the vasocontractile activity of endothelin. ${ }^{1,7)}$ Ide et al. ${ }^{7}$ reported that intracisternal endothelin injection at $0.6-1.2 \times 10^{-12} \mathrm{~mol} / \mathrm{kg}$ caused a biphasic BA constriction lasting for more than 24 hours in anesthetized dogs. Brain et al. ${ }^{2)}$ demonstrated the potent constrictor action of endothelin in the microvasculature by showing decreased regional blood flow after intradermal endothelin injection in rabbits.

In the present study, intracisternal injection of endothelin-1 induced a strong and long-lasting BA
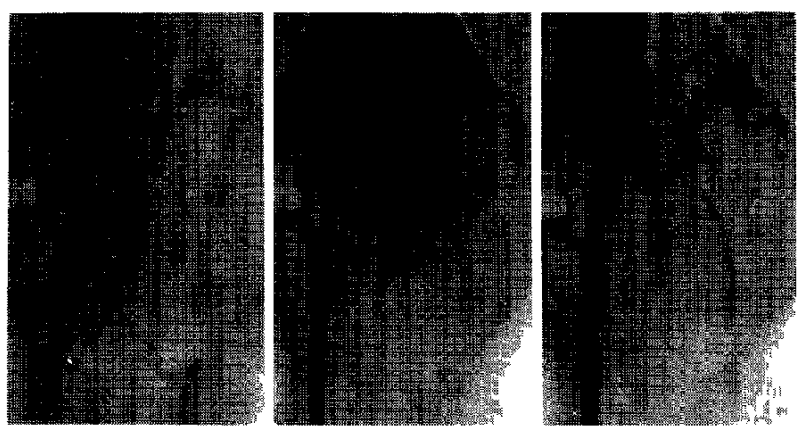

Fig. 6 Angiograms before (left), 30 minutes (center), and 60 minutes (right) after intracisternal injection of endothelin-1 $\left(10^{-9}\right.$ mol). Note severe constriction of the basilar artery, and mild to moderate constriction of the internal carotid and middle cerebral arteries. Reproduced with permission from ref. 11.

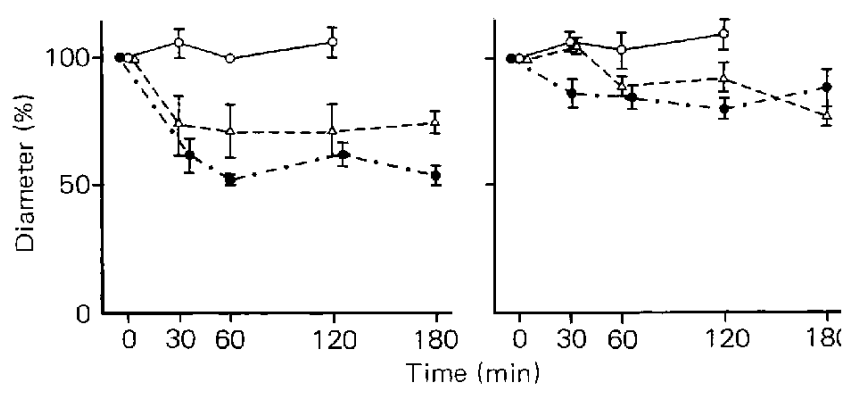

Fig. 7 Changes in the diameter of the basilar artery (left) and of the internal carotid and middle cerebral arteries (right) after intracisternal injection of endothelin-1. $\mathrm{O}$ : Group A, $\triangle$ : Group B, : Group C.

constriction. However, arterial contractions of the anterior circulation were slight to moderate compared with the BA. Since the $\mathrm{rCBF}$ measured in the present study was in the MCA territory, the flow reduction was probably not due to the ICA and MCA narrowing visualized by angiography alone. Other mechanisms such as arteriole constriction and $\mathrm{rCBF}$ regulation by the central nervous system must be considered. Autoradiography has demonstrated specific binding sites for endothelin within the circumventricular structures outside the blood-brain barrier and choroid plexus. ${ }^{101}{ }^{125}$ I-labeled endothelin was mainly absorbed in the hypothalamic and thalamic areas, lateral ventricular region, subfornical organ, globus pallidus, and caudate putamen in vitro. ${ }^{(0)}$ Other workers reported that the highest densities of ${ }^{125}$ I-labeled endothelin binding sites were in 
the granular layer of the cerebellum, choroid plexus, hippocampus, and habenular nucleus.9" Ferguson and Smith $^{\text {s) }}$ reported that microinjection of endothelin-1 into the area postrema resulted in dosedependent biphasic changes in arterial blood pressure. They considered that endothelin-1 had a specific cardiovascular action subsequent to binding within the area postrema not directly associated with the vasoconstrictor action. It is therefore possible that endothelin-1 injected into the cisterna magna was bound to the endothelin receptor in the brainstem and elicited the decrease of $\mathrm{rCBF}$ in our study.

Complete inhibition of the endothelin-induced contraction was observed in a $\mathrm{Ca}^{2+}$-free medium in vitro and marked attenuation in the presence of the $\mathrm{Ca}^{2+}$-channel blocker, nicardipine. ${ }^{21\}}$ Nicardipine $\left(10^{-8} \mathrm{M}\right)$ reverses the contraction of the canine BA induced by $10^{-8} \mathrm{M}$ endothelin, although the return to baseline requires more than 1 hour." Therefore, the influx of extracellular $\mathrm{Ca}^{2+}$ is required for the action of endothelin. Asano et al. ${ }^{11}$ reported that intracisternal injection of $10^{-11} \mathrm{~mol}$ of endothelin markedly reduced BA diameter on the first and third days in dogs, which was reversed by intravenous administration of $\mathrm{HA} 1077$, an intracellular $\mathrm{Ca}^{2-}$ antagonist. In our study, pretreatment with nicardipine inhibited the rCBF decrease caused by intracisternal injection of endothelin-1. These findings support the hypothesis that the action of endothelin-1 requires the influx of extracellular $\mathrm{Ca}^{2+}$.

Ouchi et al. ${ }^{14)}$ showed intraventricular administration of endothelin induced a dose-dependent increase in mean arterial blood pressure in conscious rats which was significantly suppressed by pretreatment with intravenous phenoxybenzamine. Moreover, intraventricular endothelin considerably increased the plasma epinephrine and norepinephrine concentrations. They concluded that endothelin had a central pressor action possibly mediated, at least partly, by catecholamine release to the peripheral vessels. In the present study, intracisternal injection of endothelin1 usually induced an increase in arterial blood pressure which was inhibited by nicardipine pretreatment. Therefore, the pressor action of endothelin-1 is also thought to be mediated by $\mathrm{Ca}^{2+}$. The relationship between intrathecal endothelin-1 administration, $\mathrm{Ca}^{2+}$ flow and catecholamine release in the systemic circulation remains unclear and requires future study.

The concentration of endothelin-1 in human plasma is $1.59 \pm 0.32 \mathrm{pg} / \mathrm{ml}^{19)}$ or $5 \mathrm{pg} / \mathrm{ml}^{.17)}$ The endothelin-1 doses administered in the present study were considerably higher. However, the endothelin sensitivity of cerebral arteries increased after experimental SAH resulting in blood-arterial wall barrier disruption to horseradish peroxidase. ${ }^{12,(8)}$ Therefore, the lower levels of endothelin-1 in the CSF may have a significant effect on rCBF after SAH in humans. Endothelin-1 may then contribute to the genesis of cerebral vasospasm.

\section{Acknowledgments}

We thank Ms. Reiko Matsuura for her excellent technical assistance. We are also indebted to Dr. Shinichi Yoshida, Department of Neurosurgery, Kanto Rosai Hospital, Kawasaki, Kanagawa, and Dr. Shinya Manaka, Department of Neurosurgery, Teikyo University Ichihara Hospital, Ichihara, Chiba, for reviewing the manuscripts, useful discussions, and statistical consultation.

This study was supported in part by scientific research fund No. 01480349 from the Ministry of Education.

\section{References}

1) Asano T, Ikegaki I, Suzuki Y, Satoh S, Shibuya M: Endothelin and the production of cerebral vasospasm in dogs. Biochem Biophys Res Commun 159: 1345-1351, 1989

2) Brain SD, Tippins JR, Williams TJ: Endothelin induces potent microvascular constriction. $\mathrm{Br} J$ Pharmacol 95: 1005-1007, 1988

3) De Mey JG, Vanhoutte PM: Heterogeneous behavior of the canine arterial and venous wall. Importance of the endothelium. Circ Res 51: 439-447, 1982

4) De Mey JG, Vanhoutte PM: Anoxia and endothelium-dependent reactivity of the canine femoral artery. J Physiol (Lond) 335: 65-74, 1983

5) Ferguson AV, Smith P: Cardiovascular responses induced by endothelin microinjection into area postrema. Regul Pept 27: 75-85, 1990

6) Hanamura $T$, Shigeno $T$, Asano $T$, Mima $T$, Takakura K: Prostaglandin profiles in relation to local circulatory changes following focal cerebral ischemia in cats. Stroke 20: 803-808, 1989

7) Ide K, Yamakawa K, Nakagomi T, Sasaki T, Saito l, Kurihara H, Yoshizumi M, Yazaki Y, Takakura K: The role of endothelin in the pathogenesis of vasospasm following subarachnoid haemorrhage. Neurol Res 11: 101-104, 1989

8) Inoue $\mathrm{A}$, Yanagisawa $\mathrm{M}$, Kimura $\mathrm{S}$, Kasuya $\mathrm{Y}$, Miyauchi T, Goto K, Masaki T: The human endothelin family: Three structurally and pharmacologically distinct isopeptides predicted by three separate genes. Proc Natl Acad Sci USA 86: 2863-2867, 1989

9) Jones CR, Hiley CR, Pelton JT, Mohr M: Autoradiographic visualization of the binding sites 
for $\left[{ }^{125}\right.$ I] endothelin in rat and human brain. Neurosci Lett 97: 276-279, 1989

10) Koseki C, Imai M, Hirata $Y$, Yanagisawa M, Masaki $\mathrm{T}$ : Autoradiographic distribution in rat tissues of binding sites for endothelin: A neuropeptide? $\mathrm{Am}$ J Physiol 256: R858-R866, 1989

11) Morimoto $T$, Yoshimoto $S$, Sasaki $T$, Saito I, Takakura K: Effect of intracisternal injection of endothelin- 1 on regional cerebral blood flow in cats, in Sano K, Takakura K, Kassell NF, Sasaki T (eds): Cerebral Vasospasm, Proceedings of the IVth International Conference on Cerebral Vasospasm. Tokyo, University of Tokyo, 1990, pp 256-258

12) Nakagomi T, Yamakawa K, Ide K, Sasaki T, Saito I, Takakura K: Role of endothelin-1 in experimental cerebral vasospasm, in Sano K, Takakura K, Kassell NF, Sasaki T (eds): Cerebral Vasospasm, Proceedings of the IVth International Conference on Cerebral Vasospasm. Tokyo, University of Tokyo, 1990, pp 242-245

13) O'Brien RF, Robbins RJ, McMurtry IF: Endothelial cells in culture produce a vasoconstrictor substance. $J$ Cell Physiol 132: 263-270, 1987

14) Ouchi $Y$, Kim S, Souza AC, Iijima S, Hattori A, Orimo H, Yoshizumi M, Kurihara H, Yazaki Y: Central effect of endothelin on blood pressure in conscious rats. Am J Physiol 256: H1747-H1751, 1989

15) Pasztor E, Symon L, Dorsch NWC, Branston NM: The hydrogen clearance method in assessment of blood flow in cortex, white matter and deep nuclei of baboons. Stroke 4: 556-567, 1973

16) Rubanyi GM, Vanhoutte PM: Hypoxia releases a vasoconstrictor substance from the canine vascular endothelium, J Physiol (Lond) 364: 45-56, 1985

17) Saito $Y$, Nakao K, Itoh H, Yamada T, Mukoyama M, Arai H, Hosoda K, Shirakami G, Suga S, Jougasaki M, Morichika S, Imura H: Endothelin in human plasma and culture medium of aortic endothelial cells: Detection and characterization with radioimmunoassay using monoclonal antibody. Biochem Biophys Res Commun 161: 320-326, 1989

18) Sasaki T, Kassell NF, Yamashita M, Fujiwara S, Zuccarello M: Barrier disruption in the major cerebral arteries following experimental subarachnoid hemorrhage. J Neurosurg 63: 433-440, 1985

19) Suzuki N, Matsumoto $H$, Kitada C, Masaki $T$, Fujino M: A sensitive sandwich-enzyme immunoassay for human endothelin. I Immunol Methods 118: $245-250,1989$

20) Tamura A, Asano $T$, Tak Y, Manaka S, Hirakawa K, Sano K: Measurement of cerebral blood flow with hydrogen clearance method: Technique and a comparative study with the venous outflow method. No To Shinkei 30: 47-54, 1978 (in Japanese)

21) Yanagisawa M, Kurihara H, Kimura S, Tomobe Y, Kobayashi M, Mitsui Y, Yazaki Y, Goto K, Masaki $T$ : A novel potent vasoconstrictor peptide produced by vascular endothelial cells. Nature 332: 411-415, 1988

Address reprint requests to: T. Morimoto, M.D., Department of Neurosurgery, Faculty of Medicine, University of Tokyo, 7-3-1 Hongo, Bunkyo-ku, Tokyo 113, Japan. 